\title{
The 8th Edition of the American Joint Committee on Cancer (AJCC8) Staging Manual: any improvement in the prognostication of oral tongue cancer?
}

\author{
Alhadi Almangush ${ }^{1,2,3}$, Tuula Salo ${ }^{1,4,5}$ \\ ${ }^{1}$ Department of Pathology, University of Helsinki, Helsinki, Finland; ${ }^{2}$ University of Turku, Institute of Biomedicine, Pathology, Turku, Finland; \\ ${ }^{3}$ Institute of Dentistry, University of Misurata, Misurata, Libya; ${ }^{4}$ Department of Oral and Maxillofacial Diseases, University of Helsinki, Helsinki, \\ Finland; ${ }^{5}$ Cancer and Translational Medicine Research Unit, Medical Research Center Oulu, University of Oulu and Oulu University Hospital, \\ Oulu, Finland \\ Correspondence to: Alhadi Almangush. Department of Pathology, University of Helsinki, Helsinki, Finland. Email: alhadi.almangush@helsinki.fi. \\ Comment on: Mascitti M, Rubini C, De Michele F, et al. American Joint Committee on Cancer staging system 7th edition versus 8th edition: any \\ improvement for patients with squamous cell carcinoma of the tongue? Oral Surg Oral Med Oral Pathol Oral Radiol 2018;126:415-23.
}

Submitted Dec 23, 2018. Accepted for publication Dec 26, 2018.

doi: $10.21037 /$ cco.2018.12.04

View this article at: http://dx.doi.org/10.21037/cco.2018.12.04

Oral tongue squamous cell carcinoma (OTSCC) is the most common carcinoma of the oral cavity, and according to a recent analysis of the Surveillance, Epidemiology, and End Results (SEER) database, patients with OTSCC are more likely to experience cause-specific mortality than patients with SCC of the other oral subsites (1). The traditional tumor $(\mathrm{T})$, lymph node $(\mathrm{N})$ and metastasis $(\mathrm{M})$ staging system has been the cornerstone for clinical classification of OTSCC, although it has been widely criticized for its low prognostic value, especially in early-stage cases $(2,3)$. Recently, the 8th edition of the American Joint Committee on Cancer (AJCC8) staging manual has been released (4), and it includes numerous changes and modifications of the TNM system that differ from the previous 7 th edition (AJCC7). For OTSCC, the most significant change in the 8 th edition was in the tumor $(\mathrm{T})$ category by incorporation of depth of invasion (DOI). In addition, extranodal extension (ENE) was added to the lymph node category $(\mathrm{N})$.

Since the changes in the AJCC8 will influence clinical decision-making, it is necessary to evaluate the prognostic implementation of the newly introduced TNM staging. In this scenario, retrospective comparative studies have been conducted to determine whether the 8th edition TNM staging showed any improvement in the classification and prognostication over the 7 th edition. Mascitti and co-authors (5) investigated the prognostic value of the AJCC7 vs. the AJCC8 staging system in 73 patients with
OTSCC. According to the evaluation with AJCC8, 25 cases were reclassified, and in particular, the use of ENE in $\mathrm{pN}$ classification identified patients with worse diseasefree survival and overall survival $(\mathrm{P}<0.05)$. We also recently re-classified our multicenter cohort of 311 early-stage cases (cT1-2N0, based on the 7th edition) according to the AJCC8, and compared the prognostic capacity of both systems (6). We noted that many cases were upstaged (even from cT1 to cT3) and were associated with worse diseasespecific survival and disease-free survival (6). Similarly, in a cohort of 298 OSCC cases, upstaging was common in $\mathrm{pT}$ and in $\mathrm{pN}$ classifications, and those upstaged cases associated with poorer prognosis (7). Moeckelmann et al. (8), in their cohort of 663 OSCC cases, were critical of the AJCC8 staging system, noting that it is more complex to use than AJCC7 due to the inclusion of DOI and ENE. However, they showed that AJCC8 stratified the survival of the patients better than the previous staging (8). These researchers also found that prognostic implementation of AJCC8 staging of OSCC was similar regardless of whether thickness of the tumor or DOI was used for T-stage (9).

The assessment of DOI can be analyzed already from the hematoxylin and eosin stained biopsies in cases where they are deep enough and taken from the most representative tumor area. Alternatively, using ultrasonography, tumor thickness is possible to evaluate preoperatively (10). In our opinion, the incorporation of the third dimension (depth or 
thickness) in the T-category is very important as it assesses the clinical behavior through the measurement of the amount of tumor infiltration. Importantly, incorporation of DOI will cause a striking difference in the staging of many cases. For example, a superficial tumor with minimum dimension $(\leq 2 \mathrm{~cm})$ was considered as T1 similar to a deep tumor $(>10 \mathrm{~mm})$ with the same diameter $(\leq 2 \mathrm{~cm})$ according the AJCC7. Now, in the AJCC8, the superficial tumor is classified as T1, while the deep tumor $(>10 \mathrm{~mm})$ is classified as T3, although both have the same dimension of $\leq 2 \mathrm{~cm}$. This remarkable change in the classification category of small OTSCC was noted in our recent study (6).

For decades, revealing the most informative parameters in OSCC staging has been a focus of research in order to improve the prognostic value of the TNM classification. The improved clinical relevance of the current AJCC8, compared to AJCC7, is a big step towards separating more precisely the low- and high-risk OSCC cases. The recent studies on OSCC from different geographic regions indicated that the prognostic significances of the criteria released in the AJCC8 staging manual are better than in the AJCC7 (5-8). Further international multicenter studies applying the AJCC8, possibly with some additional practical parameters, should be conducted in order to potentially further improve the prognostic value of OTSCC/OSCC patients using TNM staging.

\section{Acknowledgments}

None.

\section{Footnote}

Conflicts of Interest: The authors have no conflicts of interest to declare.

\section{References}

1. Farhood Z, Simpson M, Ward GM, et al. Does anatomic subsite influence oral cavity cancer mortality? A SEER database analysis. Laryngoscope 2019;129:1400-6.

2. Almangush A, Bello IO, Coletta RD, et al. For early-stage oral tongue cancer, depth of invasion and worst pattern of invasion are the strongest pathological predictors for locoregional recurrence and mortality. Virchows Arch 2015;467:39-46.

3. Piazza C, Montalto N, Paderno A, et al. Is it time to incorporate 'depth of infiltration' in the T staging of oral tongue and floor of mouth cancer? Curr Opin Otolaryngol Head Neck Surg 2014;22:81-9.

4. Amin MB, Edge S, Greene F, et al. AJCC Cancer Staging Manual, 8th edition. New York: Springer, 2017.

5. Mascitti M, Rubini C, De Michele F, et al. American Joint Committee on Cancer staging system 7th edition versus 8th edition: any improvement for patients with squamous cell carcinoma of the tongue? Oral Surg Oral Med Oral Pathol Oral Radiol 2018;126:415-23.

6. Almangush A, Makitie AA, Makinen LK, et al. Small oral tongue cancers $(</=4 \mathrm{~cm}$ in diameter $)$ with clinically negative neck: from the 7th to the 8th edition of the American Joint Committee on Cancer. Virchows Arch 2018;473:481-7.

7. Matos LL, Dedivitis RA, Kulcsar MAV, et al. External validation of the AJCC Cancer Staging Manual, 8th edition, in an independent cohort of oral cancer patients. Oral Oncol 2017;71:47-53.

8. Moeckelmann N, Ebrahimi A, Tou YK, et al. Prognostic implications of the 8th edition American Joint Committee on Cancer (AJCC) staging system in oral cavity squamous cell carcinoma. Oral Oncol 2018;85:82-6.

9. Dirven R, Ebrahimi A, Moeckelmann N, et al. Tumor thickness versus depth of invasion - Analysis of the 8th edition American Joint Committee on Cancer Staging for oral cancer. Oral Oncol 2017;74:30-3.

10. Tarabichi O, Bulbul MG, Kanumuri VV, et al. Utility of intraoral ultrasound in managing oral tongue squamous cell carcinoma: Systematic review. Laryngoscope 2019;129:662-70.
Cite this article as: Almangush A, Salo T. The 8th Edition of the American Joint Committee on Cancer (AJCC8) Staging Manual: any improvement in the prognostication of oral tongue cancer? Chin Clin Oncol 2019;8(Suppl 1):S8. doi: 10.21037/ cco.2018.12.04 